\title{
An Analysis of the Allometric and Multifractal Features of a Development in the Urban-Rural Area in the Lower Reaches of the Yangtze River: 2012 Cross-Sectional Data of Four Provinces and One City
}

\author{
Zhi-jun Song, ${ }^{1,2}$ Yi Chen $\left(\mathbb{D},{ }^{3}\right.$ Yun Li ${ }^{3},{ }^{3}$ and Xin Zhao ${ }^{4}$ \\ ${ }^{1}$ The College of Environment and Planning, Henan University, Henan, Kaifeng 475001, China \\ ${ }^{2}$ Key Laboratory of Geospatial Technology for the Middle and Lower Yellow River Regions (Henan University), \\ Ministry of Education, Henan University, Henan, Kaifeng 475001, China \\ ${ }^{3}$ Industry 4.0 Artificial Intelligence Laboratory, Dongguan University of Technology, Dongguan 523808, China \\ ${ }^{4}$ College of Environmental Science and Engineering, Beijing Forestry University, Beijing 100083, China
}

Correspondence should be addressed to Yi Chen; leo.chen.yi@live.co.uk and Yun Li; yun.li@ieee.org

Received 13 November 2019; Accepted 10 March 2020; Published 13 May 2020

Academic Editor: Qingling Wang

Copyright (C) 2020 Zhi-jun Song et al. This is an open access article distributed under the Creative Commons Attribution License, which permits unrestricted use, distribution, and reproduction in any medium, provided the original work is properly cited.

\begin{abstract}
The provinces of Jiangsu, Anhui, Jiangxi, and Zhejiang and the city of Shanghai in the lower reaches of the Yangtze River forming a concentrated distribution area of developed cities and rich villages in China are so called "the four provinces and one city," which have experienced rapid and typical urban-rural development. By drawing on the perspective of allometric growth, this paper conducts fundamental analysis and multifractal analysis of 52 prefecture-level cities in four provinces and one city in the lower reaches of the Yangtze River. Fundamental analysis shows that there are different patterns of allometric growth in various areas of the region, as well as the emergence of diffusion in fundamental activities and of polarisation in advanced activities. Multifractal analysis also shows that the comprehensive regional development is dominated by large- and medium-sized cities, but various fields have different fractal spectrums and form six interrelated urban-rural integration modes. The multiple spatial dissymmetry disclosed in the analysis also shows that each field had its own allometric growth features during the regional development. This paper adopts the fitting function and the relevant parameters of the fractal dimension to quantify the evolution in particular fields. Analysis shows that the developments in prefecture-level cities are different and represent the regional evolution status; the urbanrural development in metropolises and small- and medium-sized cities is nonmainstream.
\end{abstract}

\section{Introduction}

The four provinces and one city (the provinces of Jiangsu, Anhui, Jiangxi, and Zhejiang and the city of Shanghai) in the lower reaches of the Yangtze River form a concentrated distribution area of developed cities and rich villages in China; this area has experienced rapid and typical urbanrural development. The area comprises approximately $517,600 \mathrm{~km}^{2}$ and accounts for only $5.41 \%$ of the total area of China; however, its gross domestic product (GDP) in 2012 accounted for $24.58 \%$ of the national GDP (approximately 14,110 billion yuan), and the number of workers in the region accounted for $22.09 \%$ of the total number of workers in China (approximately 29.56 million). Meanwhile, the development in various areas of the region has been different, and multidisciplinary research shows the following. (1) Various development functions radiate, deescalate, and transfer from the centre of the region (Shanghai) to the periphery (Northern Jiangsu Province, Western Anhui Province, and Southern Jiangxi Province), which reflects the corresponding spatial-temporal coupling status [1-5]. (2) Although there has been varied urban-rural development in this region, the villages and their nearby towns are closely related; in addition, because of the administrative division, 
the prefecture-level cities and towns in the lower reaches of the Yangtze River often constitute a relatively unified geographical complex $[6,7]$. Accordingly, the regional urbanrural landscapes have obvious features of prefecture-level cities. (3) In the lower reaches of the Yangtze River, there is an international city (Shanghai) and unenlightened villages (the peripheral rural area). Therefore, the types of urbanrural integration at the prefecture level are diversified and interact with one another [8-10]. (4) There is a strong continuity in the external spatial changes of the urban-rural compound landscapes, and the development level deescalates from the centre of the region to the periphery $[6,7,11,12]$. Many spatial dissymmetries at various development levels emerge in various fields.

This study aims to perform the fundamental research on the allometric growth modelling to reveal regional development mechanism. To disclose the real situation of the urban-rural development in the four provinces and one city in the lower reaches of the Yangtze River (52 cities including prefecture-level cities, capital cities, and a municipality, which are abbreviated to prefecture-level cities), this paper draws on a spatial autocorrelation analysis and then adopts the perspective of allometric growth and multifractal techniques to quantify the types and features of the urbanrural integration and complexity of the spatial evolution. The contributions of this work are the following: (1) multifractal analysis of 52 prefecture-level cities, (2) algometric growth features during the regional development, (3) a quantitative understanding and graphical representation of spatial autocorrelation and multifractal analysis, and (4) including the urban-rural developments in metropolis and small- and medium-sized cities. Theoretically, this manifests itself as a study of the spatial autocorrelation, allometric growth, and multiple fractal features in the lower reaches of the Yangtze River, as well as the comparison and correlation of the three evolutionary features. The purpose is to analyse the spatial representation and evolution mechanism, and three fused perspectives of spatial evolution details show the whole picture of the spatial development of the region.

The rest of the paper is organised as follows. Following this Introduction section, Section 2 introduces the method and data in the empirical analysis. Section 3 presents the fundamental study on the regional evolution using spatial autocorrelation analysis. Section 4 performs the spatial multifractal analysis. Section 5 presents the regional allometric growth analysis and multifractal analysis. Section 6 carries out the discussion and Section 7 presents a brief conclusion and the potential future research.

\section{Methods and Data}

2.1. Perspective of Allometric Growth and Multifractal Techniques. The development of urban and rural areas in the lower reaches of the Yangtze River is a complex process. The nonagricultural sector has always been developing at a nonnegative pace, while the rural areas have gradually shrunk and have provided ample space for allometric growth in other areas. Therefore, the local cities themselves are an urban-rural complex that integrates homogenized villages with increasingly heterogeneous towns [4, 13, 14, 15]. Correspondingly, there are three main technical methods in this study: GIS-based spatial autocorrelation analysis, allometric growth analysis, and multifractal analysis (strictly speaking, single fractal is only a special case of multifractal). The logical relationship between them can be expressed as follows: GIS-based spatial autocorrelation is an external manifestation of allometric phenomena and can show this kind of spatial complexity in the form of an icon; it is intuitive, but the complexity of the mathematical theory Parsing (depth analysis) is not sufficient. Therefore, on the basis of spatial autocorrelation analysis, allometric growth analysis can further reflect the internal driving factors of regional spatial evolution and the overall background of development. The allocating of various fields in space will inevitably leave "traces" such as hierarchy of spatial attributes and scales of various types of land types. Such traces can often be multifractal. Several indicators of single fractal case are reflected. Therefore, a comprehensive analysis based on the combination of the three will help reveal the macrodevelopment mechanism and microspecific evolution "track" of the integration of urban and rural areas in the lower reaches of the Yangtze River.

Regional space is scale-free. Conventional mathematical methods are successful in describing the development with characteristic lengths, but they cannot describe the real situation of a region without a characteristic scale. Fractal geometry and allometric growth theory are effective tools to analyse the characteristic of being scale-free.

Allometric analysis is devoted to the nonnegative proportional relationship between two elements of a geographical system or one element and the entire system [16-19]. Spatial regions are typical complex systems where various fields are differently related and evolve differently. Currently, nonagricultural industrialisation, urbanisation, and industrialisation drive the stronger growth-oriented development in many fields (such as urban construction and the tertiary industry) in China. However, development in other fields, for example, traditional agriculture, is weakening and declining. Although their evolution is not allometric because of their declining development, these other fields gradually acquire spatial fractal features and provide broad space for the fields with stronger development. These two types of development are intertwined and form a complex regional system; the allometric growth that is generated in the fields with stronger development dominates the development of the region. In reality, the development of allometric growth in the multifields has two distinct features. (1) The allometric growth of a field follows the following law: the breaking of the original equilibrium $\longrightarrow$ the emergence of spatial autocorrelation $\longrightarrow$ the emergence of trends $\longrightarrow$ the formation of spatial self-organisation (e.g., fractal) [20]. (2) The complex of various allometric growths facilitates the overall evolution of the region, but the development of fields is different, and the difference results in the asynchronous evolution in various fields [16].

Corresponding to the perspective of allometric growth, the multifractal analysis is an important method to analyse the regional allometric growth in economic geography. 
Compared to the inability of conventional technology to conduct research on spatial self-organisation, the multifractal analysis is successful in analysing spatial complexity and providing a quantitative evaluation for the spatial layout and evolutionary processes. The main criteria of multifractal features are as follows: (1) the main fractal dimension values, which reflect the noninteger space of the fractal from a different perspective; (2) the spectrum width, because when the range of the multifractal spectrum is wider, the fractal is more complex; (3) the spectrum truncation, because the truncation of the multifractal dimension spectrum indicates the main fluctuation range of the changes in the fractal; (4) the shape of the fractal spectrum, which reflects the regularity of the changes in the self-organisation of the reaction system; and (5) the peak of the fractal dimension, which reflects the dominant components of system changes $[21,22]$.

The development of the urban-rural area in the lower reaches of the Yangtze River is a complex process, and the nonagricultural fields have been developing at a nonnegative growth rate. The agricultural area has gradually shrunk and provided broad space for allometric growth in other fields. Therefore, the prefecture-level city itself is an urban-rural complex that comprises homogenised villages and heterogenised towns $[13,14,23]$. In this sense, analysis of allometric growth and its multifractal features of urban-rural integration in the lower reaches of the Yangtze River is devoted to examining the phenomenon of the diffusion of urban functions from the heterogeneous region (Yangtze River Delta) to the homogeneous rural peripheral area.

2.2. Data. The statistical data are derived from the China Urban Statistical Yearbook and the China Regional Economic Statistical Yearbook [24, 25]. The spatial data are derived from Landsat TM and ETM remote sensing images and related thematic maps; this research constructs the corresponding spatial database on ArcGIS 10.1 and the GeoDa software platform. The urban-rural developments on the same prefecture level are highly correlated, and the urban-rural data are not listed; the statistical data of prefecture-level cities can be used to represent the corresponding development of the urban-rural integration. In this case, the object of analysis is the regional complex of the urban-rural area (or urban-rural complex-prefecture-level cities). Thus, the spatial evolution of the urban-rural integration in the lower reaches of the Yangtze River can be understood as prefecture-level cities that show urban-rural composite landscapes.

Therefore, according to the law of regional evolution, the breaking of the original equilibrium $\longrightarrow$ the emergence of spatial autocorrelation $\longrightarrow$ the emergence of trends $\longrightarrow$ the formation of spatial self-organisation (e.g., fractal). Section 2.1 reports a fundamental mathematical analysis to reveal the equilibrium of the multifields development in the lower reaches of the Yangtze River and the patterns of spatial evolution. Section 2.2 adopts GIS techniques to demonstrate the spatial autocorrelation in various fields of the region and verifies the conclusions that are drawn in Section 2.1. In
Section 3, this research analyses spatial self-organisation, which is represented by multifractals. Additionally, based on the fractal dimension, Section 3 reveals the evolution details of some fields with the multifractal features. Drawing on the thoughts of multiple spatial dissymmetries, Section 4 explores the reason behind the features of the mathematical law and the complexity (such as multifractal features) that is found in the development of the lower reaches of the Yangtze River.

\section{Fundamental Analysis}

3.1. Fundamental Mathematical Analysis of Regional Evolution. The power function is suitable for describing the correlation of multifields in a region (e.g., allometric growth), but it is often transformed into an exponential function, a logarithmic function, or other types of functions [20]. Accordingly, this paper conducts a fundamental mathematical analysis of the development in various fields in 52 prefecture-level cities in the lower reaches of the Yangtze River.

This paper performs function fitting on the built-up areas in 52 prefecture-level cities in the lower reaches of the Yangtze River (the regional urban-rural integration is normally dominated by the attributes of the built-up areas). The results show that the hierarchical distribution is more statistically significant with the fitting of the power function (the goodness of fit is $R^{2}=0.9677$ ), and $R^{2}$ of the 40 cities where the built-up area is more than $60 \mathrm{~km}^{2}$ is as high as 0.9884, as shown in Figure 1(a). Based on the results, one can conclude that the cities that agglomerate the socioeconomic activities of the four provinces and one city have the features of spatial complexity and that the foundation of urban-rural development will be transformed through functional changes to businesses, services, industries, and daily living. The analysis also shows the following:

(1) Each field will adapt to the external environment, making the regional development more complex. For example, the exponential function is more suitable for describing the GDP per capita in the 52 prefectural-level cities $\left(R^{2}=0.9829\right)$ than is the power function $\left(R^{2}=0.7965\right)$, as shown in Figure 1(b); this indicates that, compared with the power law evolution, the production activities in the lower reaches of the Yangtze River tend to have progressive spatial changes (the fitting curve of the exponential function is gentler). The results of the mathematical analysis reflect the influence of the national industrial layout.

(2) The level ranges of prefecture-level cities with the feature of self-organisation in different fields are not the same. For example, the results of the top 33 cities that are ranked by the average salary in the municipal districts are more statistically significant with the fitting of the power function $\left(R^{2}=0.9469\right.$, close to $0.95)$ than are the results of all the cities; the goodness of fit, $R^{2}(0.8643)$, is well below the threshold of 0.95, as shown in Figure 1(c). 


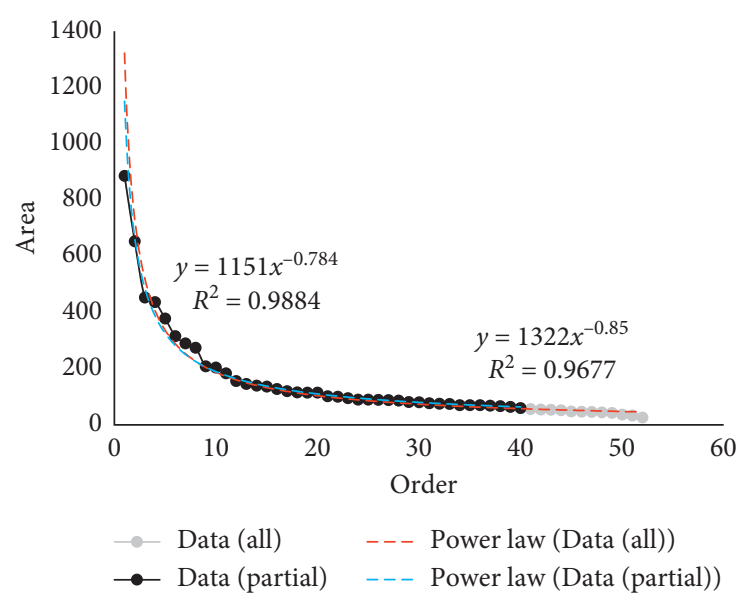

(a)

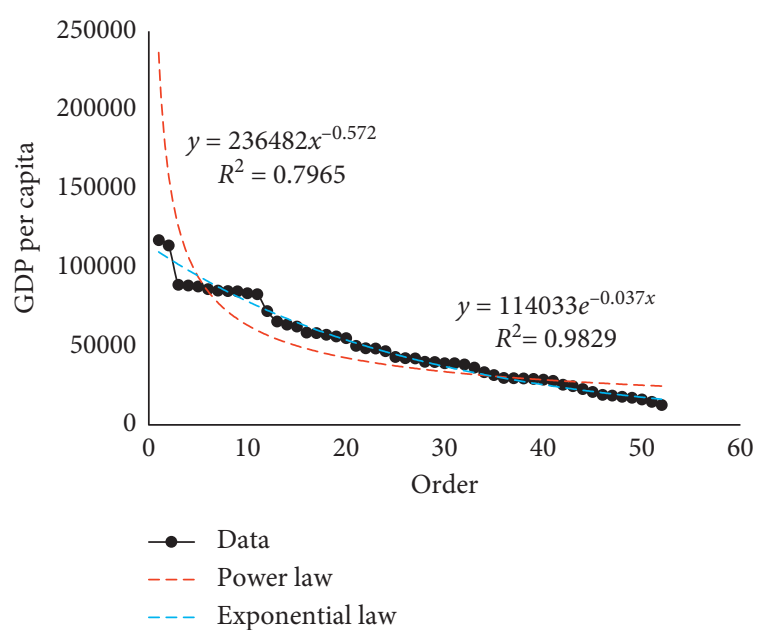

(b)

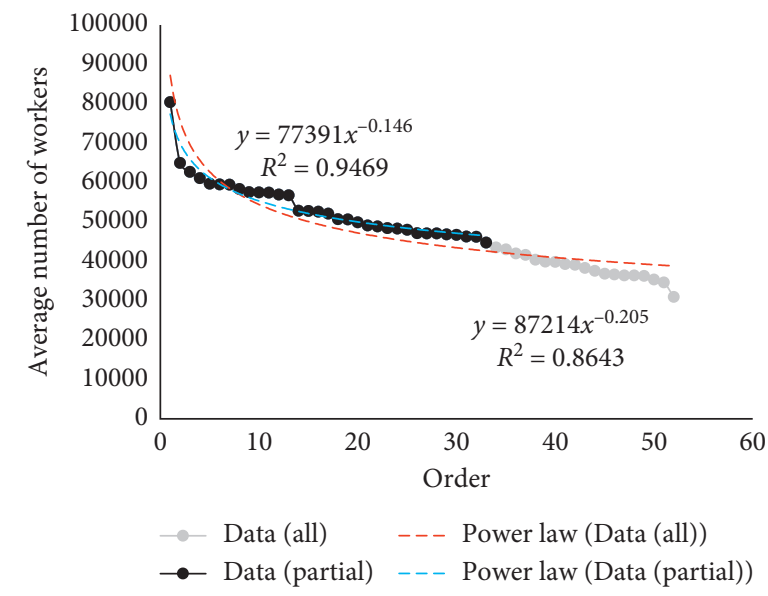

(c)

FIGURE 1: Function fitting of the Yangtze river's lower reaches. (a) Built-up area. (b) GDP per capita. (c) Average salary in the municipal districts.

(3) Microscale/fundamental socioeconomic activities tend to diffuse to small- and medium-sized cities, while large- and medium-sized cities tend to agglomerate meso-macro-scale/advanced activities. According to the indicators mentioned above, the analysis shows that the slope of the exponential function-fitting curve of the GDP per capita is smaller than the slope of the power function-fitting curve of the built-in areas; and $R^{2}$ of the sample size of 50 cities is more statistically significant than $R^{2}$ of the top 33 cities. These results show that microscale socioeconomic activities have been coupled with grassroots production and living activities and have made their diffusion fit the exponential function. Moreover, the power law evolution of the built-up areas emphasises the agglomeration of the power source of the urban-rural integration (large- and medium-sized cities).

Therefore, the development of 52 prefecture-level cities in the lower reaches of the Yangtze River has broken the homogenisation of the region and shows the trend of the law of evolution, and the law of evolution can be described by corresponding mathematical functions. Meanwhile, these mathematical functions emphasise the complexity of the development in this region. Points 1 and 2 indicate the nonequilibrium and diversity of the spatial development in the lower reaches of the Yangtze River. Point 3 indicates that there will be varied allometric growth in various fields of this region. Regression analysis of a variety of data demonstrates the universality of the abovementioned phenomena, and the phenomena are proven in the follow-up analysis.

3.2. Regional Spatial Autocorrelation Analysis. Spatial autocorrelation analysis is an intuitive understanding of the changes to the self-organisation of the regional entity space (the regularity revealed by the above analysis) [26]; it is the foundation of the in-depth theoretical/mathematical analysis (Section 3).

3.2.1. Global Spatial Autocorrelation Analysis. Global spatial autocorrelation analysis of 12 fields in 52 prefecture-level 
cities (agricultural data excluded) (Table 1) shows that, at the 95\% confidence interval, 10 indicators, including GDP (Geary's index $C$ is 0.726 ), total salary (0.731), population density $(0.746)$, the average salary in the municipal districts (0.729), and the average number of workers (0.753), are statistically significant. The results indicate that the 10 nonagricultural fields are positively spatially correlated. Generally, the values of the attributes mentioned above have declined from the centre of the region (Yangtze River Delta) to the periphery area, and these 10 fields carry out scale expansion, spatial expansion, and transformation in the lower reaches of the Yangtze River.

There is no significant spatial autocorrelation between the indicators of the built-up areas and the population density in municipal districts. These two indicators are not statistically significant because the government's macroscale control (such as urban planning, industrial planning, social security, and reemployment opportunities) offsets the polarised effects of the corresponding activities so that they have not formed any significant agglomeration or diffusion centres.

3.2.2. Local Spatial Autocorrelation Analysis. This paper adopts the Lisa index to evaluate the spatial autocorrelation of 52 prefecture-level cities (Table 2 and Figure 2), and the results show the following:

(1) There is an obvious spatial polarisation-diffusion effect on the entire region, and the level of development has declined from the Yangtze River Delta to the west of Anhui Province, the west of Jiangxi Province, and the south of Anhui Province.

(2) Shanghai and Taizhou in the east of Zhejiang Province are high isolation centres.

(3) The development of Wenzhou is a unique mode of urban-rural development and exhibits of the "Greater Shanghai Economic Circle."

(4) The focus of economic development has shifted to Jiaxing, which is adjacent to Shanghai, and there is spatial dissymmetry between Jiaxing and the economic centre, Shanghai.

(5) By various indicators, high isolation centres emerge in the peripheral areas (such as Nanchang, Huainan, and Xuzhou).

Generally, points 4 and 5 reflect that there are multifields with spatial dissymmetry and the dissymmetry of development in the lower reaches of the Yangtze River, indicating that heterogeneity is the normal state of the regional development. Points 1,2 , and 3 reveal the external spatial appearance of the region, which is further analysed in Section 3.

It is worth noting that only three indicators (the average salary in the municipal districts, the GDP per capita, and the urban built-up area) are statistically significant in the global spatial autocorrelation analysis. The first two indicators are statistically significant in the global spatial autocorrelation test; the third indicator is statistically significant in the case of lower requirements. The three indicators represent the typical attributes of the micro-meso-macro-scale levels of socioeconomic activities.

3.2.3. Allometric Growth Analysis. After the graphical spatial correlation shown in Section 3.2, a theoretical allometric analysis should be performed. For a regional spatial system with allometric growth, the power-law relationship should be satisfied between each component (specifically, this is the development area), so that the entire space system can have a generalised fractal nature. Furthermore, we can establish an allometric growth matrix based on the power law relationship to determine the behaviour (relative evolution or relative decline) of this spatially allometric system and its components, so as to analyse and evaluate the development of the entire region and components $[15,18,19]$. However, through the analysis of the fitting of the two indicators (see Figure 3 for an example) and Section 3.1, we can see the following: (1) in reality, many areas in the lower reaches of the Yangtze River are more scientifically described by other functions and do not satisfy the power law; (2) not all prefecture-level cities can cover a unified allometric system. So we cannot establish a basic allometric growth matrix.

A following-up question has been brought out: Is there an allometric growth in the lower reaches of the Yangtze River? In fact, (1) the spatial autocorrelation analysis of 3.2 has shown that the area has a basis for allometric growth, but allocating space (or the number of prefecture-level cities included) in each area may be different; (2) space complex systems are generally in nonstatic idealized systems, the transition between states should be the norm of its existence, and the transition of power law is also objective; (3) generally, the long-term development of developed regions will have different degrees of allometric growth [27]. Therefore, we do not rule out the existence of allometric growth in the case where the scope of this analysis is not accurate enough for each development field and the system is not an ideal quasi-static system. Moreover, we can also appreciate the existence of this allometric growth phenomenon through the evaluation values of the various fields of development quantified in Section 4 and the spatial misalignment between them. Therefore, the allometric idea will be accompanied by the description of the full text and eventually form a unified conclusion with other analyses (see Section 5).

3.3. Preliminary Understanding. The analysis in Section 2.1 shows that there is nonequilibrium and multiple spatial dissymmetries in the lower reaches of the Yangtze River and regularity in the spatial and hierarchical patterns (Table 2 and Figure 2). The analysis in Section 2.2 contributes to three important indicators that represent the micro-meso-macroscale levels of development (the average salary in the municipal districts, the GDP per capita, and the urban built-up area). Based on previous researches $[1-3,5-7,28-31]$, this paper concludes that the multifields development in the lower reaches of the Yangtze River tends to be statistically significant and spatially autocorrelated. 
TABLE 1: Results of the global spatial autocorrelation analysis of four provinces and one city in the lower reaches of the Yangtze River.

\begin{tabular}{lccccccccccccccc}
\hline & GDP & $\begin{array}{c}\text { GDP } \\
\text { per } \\
\text { capita }\end{array}$ & Area & Population density & $\begin{array}{c}\text { Average number of } \\
\text { workers }\end{array}$ & \multicolumn{2}{c}{$\begin{array}{c}\text { Total salary of } \\
\text { workers }\end{array}$} & Average salary \\
& City & City & City & $\begin{array}{c}\text { Built- } \\
\text { up area }\end{array}$ & City & $\begin{array}{c}\text { Municipal } \\
\text { district }\end{array}$ & City & $\begin{array}{c}\text { Municipal } \\
\text { district }\end{array}$ & $\begin{array}{c}\text { City } \\
\text { Municipal } \\
\text { district }\end{array}$ & $\begin{array}{c}\text { City } \\
\text { Municipal } \\
\text { district }\end{array}$ \\
\hline $\begin{array}{l}\text { Geary's } C \\
\begin{array}{l}\text { Significance } \\
\text { level }(0.05)\end{array}\end{array}$ & 0.7262 & 0.7844 & 0.8668 & 0.9048 & 0.7459 & 1.0520 & 0.7867 & 0.7529 & 0.7308 & 0.7566 & 0.7296 & 0.7287 \\
\hline
\end{tabular}

Based on Tables 1 and 2 and Figure 2, the spatial layout of the urban-rural integration modes in the lower reaches of the Yangtze River can be summarised as follows:

(1) The urbanisation mode represented by the development pattern of Shanghai is a typical pattern of urban-rural integration that promotes suburban industrialisation and urbanisation by expanding built-up areas.

(2) The pattern of the urban-rural integration of the Yangtze River Delta mode with the higher level of development mode (the south of Jiangsu Province mode), including the south of Jiangsu Province and the north of Zhejiang Province, is formed by promoting nonagricultural industrialisation in the suburbs with the development of township enterprises.

(3) The pattern of the south of Zhejiang Province mode (Wenzhou mode) promotes regional development through the development of the individual and private economy.

(4) The transitional mode in the central area actively promotes the optimisation of the industrial structure in prefecture-level cities by promoting the development of industries, businesses, services, and new agriculture.

(5) The polarisation mode in the central large- and medium-sized cities (such as Nanchang and Xuzhou) is an integrated development mode; its main manifestation is the dissymmetry between the developed cities and underdeveloped hinterland.

(6) The goal of the traditional mode in the peripheral area (the west and south of Anhui Province and the west and east of Jiangxi Province) is to solve the issues of agriculture, farmer, and rural areas and to enhance the development strength of prefecturelevel cities.

The above understanding is consistent with people's intuitive feelings and the relevant research results. However, spatial autocorrelation analysis (Section 2.2) can only reflect the external manifestation of the region. The corresponding mathematical analyses (Section 2.1) can contribute some theoretical support, but they cannot explain the reasoning behind the phenomena because they cannot reflect the complex relationship among the modes listed above or explain their spatial self-organisation behaviour (e.g., multifractal).

\section{Spatial Multifractal Analysis}

4.1. MATLAB-Based Analysis and Criterion. Based on the preliminary understanding established in Section 3.2, this paper conducts a multifractal analysis of the comprehensive indicator of prefecture-level cities (the scale of GDP) and the three typical indicators that were filtered in Section 3.2.2 to reveal the features and mechanism of the multifields' evolution in the lower reaches of the Yangtze River [13, 21].

Using the MATLAB toolbox-multifractal detrended fluctuation analysis (MFDFA), in the case of sorting the indicator data in descending order, the MATLAB-based results of the generalised fractal dimension $D_{q}$ given in equation (1), the quality index $\tau_{q}$ given in equation (2), the multifractal analysis in spectrum $h_{q}$ given in equation (3), and the singularity exponent $H_{q}$ given in equation (4) are shown in Figure 3, which indicates that the above indicators present multifractal features in 52 prefecture-level cities in the lower reaches of the Yangtze River and that the indicators are statistically significant in relevant tests. The above indicators have similar peak values and shapes of the fractal dimension and have different values of the fractal dimension, spectrum width $s$, and spectrum truncation [21, 22].

Specifically, in equation (1), the generalised fractal dimension $D_{\mathrm{q}}$ [32] can be stated in equation (1), in which $q$ denotes the order of moment $(q=\ldots,-2,-1,0,1,2, \ldots)$, and we divide the phase-space to $N(s)$ hypercubes of edge $s$; the probability to find a point in the $i$ th hypercube is denoted by $P_{i}$ :

$$
D_{q}=\frac{1}{q-1} \lim _{s \longrightarrow 0} \frac{\log \sum_{i=1}^{N(s)} P_{i}(s)}{\log s}=-\lim _{s \longrightarrow 0} \frac{I_{q}(s)}{\log s} .
$$

In equation (2), $\tau_{q}$ refers to the mass exponent of order $q$, and $D_{q}$ and $\tau_{q}$ compose the set of global parameters of multifractals sets:

$$
\tau_{q}=(q-1) D_{q}
$$

We can transform the global parameters $\tau_{q}$ and $D_{q}$ into the local parameters $H_{q}$ and $h_{q}$, as shown in the following equations: 


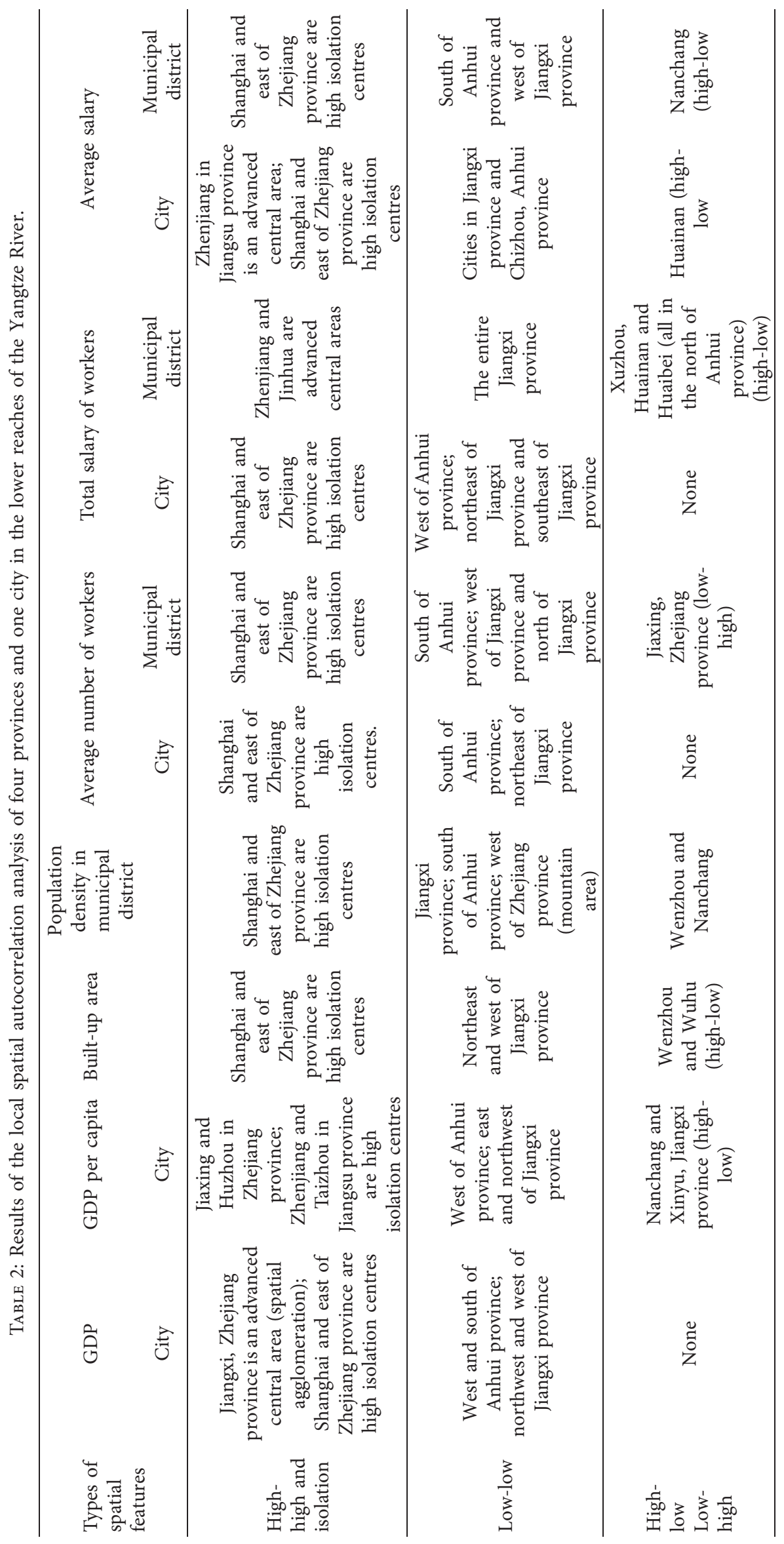




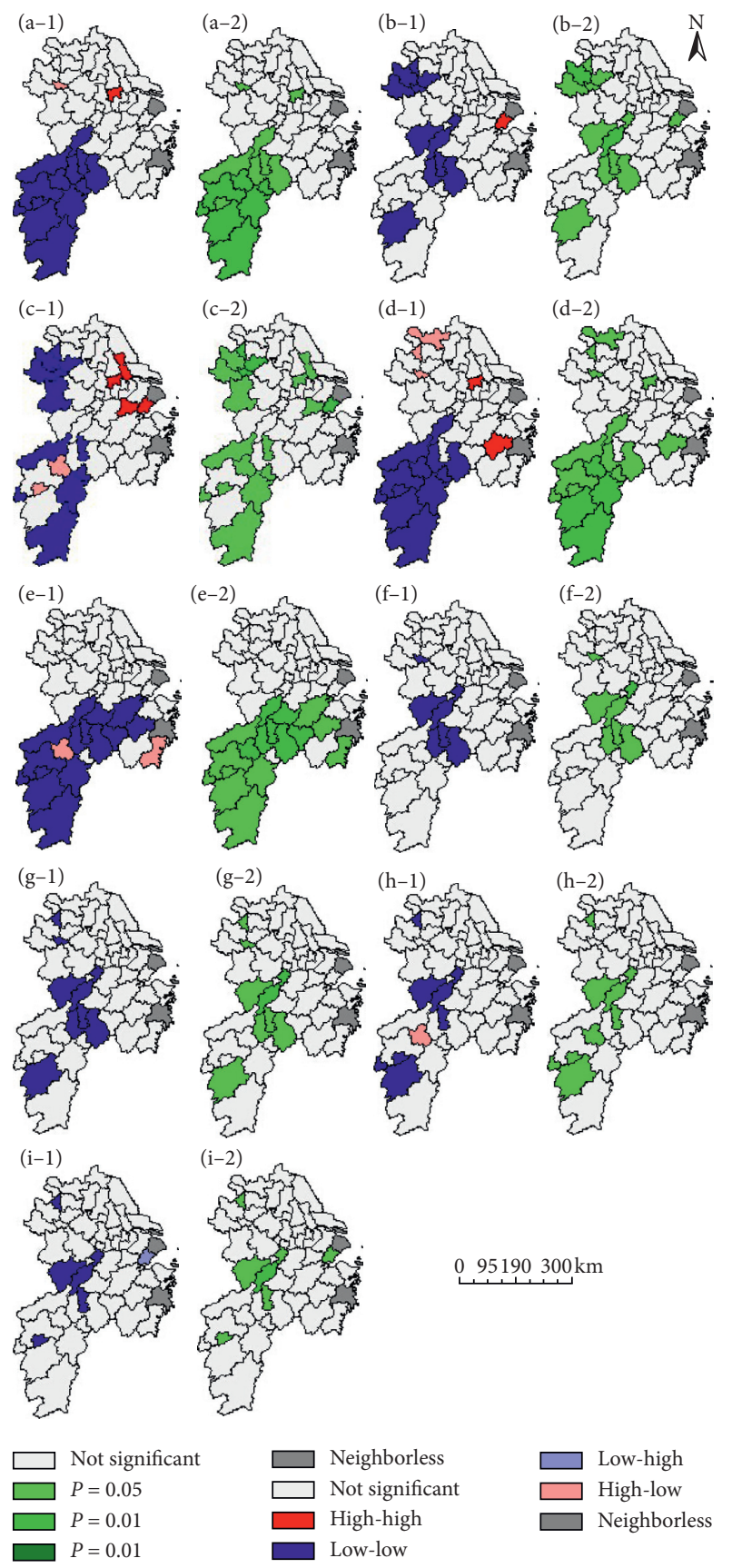

FIGURE 2: Spatial autocorrelation analysis of four provinces and one city in the lower reaches of the Yangtze river: $\left({ }^{*}-1\right)$ illustration of significance level; $\left({ }^{*}-2\right)$ illustration of spatial autocorrelation; (a) illustration of average salary; (b) illustration of GDP; (c) illustration of GDP per capita; (d) illustration of average salary in the municipal districts; (e) illustration of the built-up area per capita; (f) illustration of number of workers; ( $\mathrm{g}$ ) illustration of workers' salary; (h) illustration of worker's salary in municipal district; (i) illustration of number of workers in municipal district.

$$
\begin{aligned}
& H_{q}=D_{q}+(q-1) \frac{\mathrm{d} D_{q}}{\mathrm{~d} q}, \\
& h_{q}=q H_{q}-(q-1) D_{q} .
\end{aligned}
$$

The specific analysis shows that the comprehensive indicator (the scale of GDP) and micro-macro-scale indicators present significant multifractal features. The mesoscale indicator (built-up area) presents multifractal features by using normalised data (for this reason, the analysis process is not shown in Figure 4). Table 3 lists the capacity dimension $\left(D_{0}\right)$, information dimension $\left(D_{1}\right)$, and correlation dimension $\left(D_{2}\right)$ of the above four indicators. The capacity dimensions $\left(D_{0}\right)$ are all roughly equal to 1 , which indicates that the result is from a fractal analysis for a data sequence whose spatial dimension is approximately 1 . Accordingly, this finding indicates that the result is not from a fractal analysis for a grid fractal dimension or from an analysis for the distribution of plaques with spatial dimensions between 2 and 3 . The principles that support this conclusion can be found in Ihlen's [21] and Kantelhardt et al.'s [22] researches.

The features of the above four indicators are listed as follows:

(1) The multifractal information dimension (0.7029) and the correlation dimension (0.1480) of the GDP of 52 prefecture-level cities are between the average salary of workers in municipal districts and the GDP per capita. This result indicates that the hierarchical pattern of 52 prefecture-level cities is due to micromeso-macro-scale comprehensive development and appears to fluctuate and deescalate. The spectrum width of the multifractal (4.7048; see the curves at the lower right-hand corner of Figure 3) indicates that this indicator (GDP of 52 cities) is the most complex, which may be reflective of the synergistic effect on the regional integrated system. The multifractal of GDP shows that the strength of prefecture-level cities does not expand in a single-radiation manner; thus, the regional space that is shown in Figures 2 and 4 is becoming more complicated and does not appear to have a regular descending spatial layout.

(2) In the case of sorting the data in descending order, the information dimension $(0.8185)$ and the correlation dimension (0.4826) of the average salary in the municipal districts (on the microscale level) have the highest values; this result indicates that the average salary in the municipal districts decreases at the lowest speed from the economic centre (such as Shanghai, Nanjing, and other cities) to small- and medium-sized cities. Meanwhile, the average salary in the municipal districts is slower than the rate of decline in the GDP per capita, verifying the conclusion regarding the mathematical analysis of microscale activities which was noted in point 3 in Section 2.1. The multifractal dimension of this indicator-the average salary in the municipal districts-is the least complex (spectrum width of 1.9316) among the multifractal dimensions of the comprehensive indicator, microscale indicator, and macroscale indicator. The least complexity results from the government policy of income adjustment. 


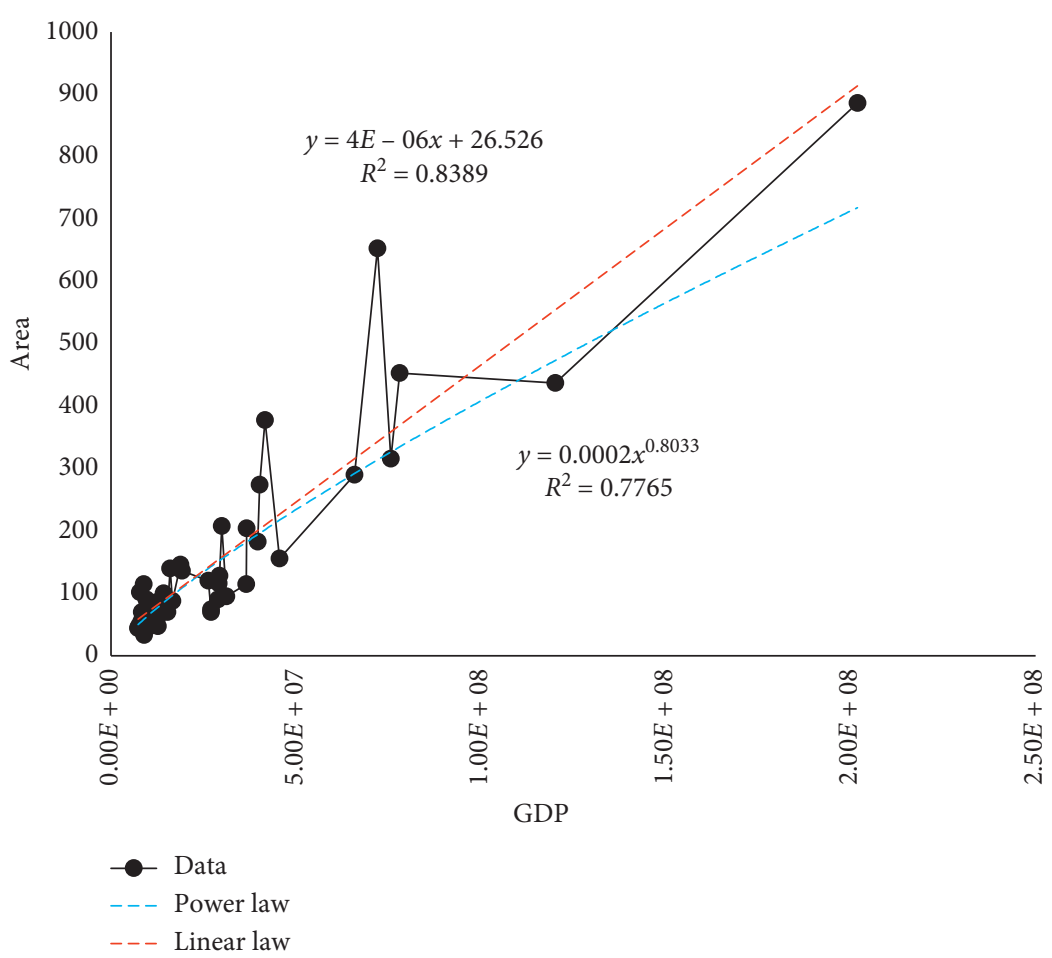

(a)

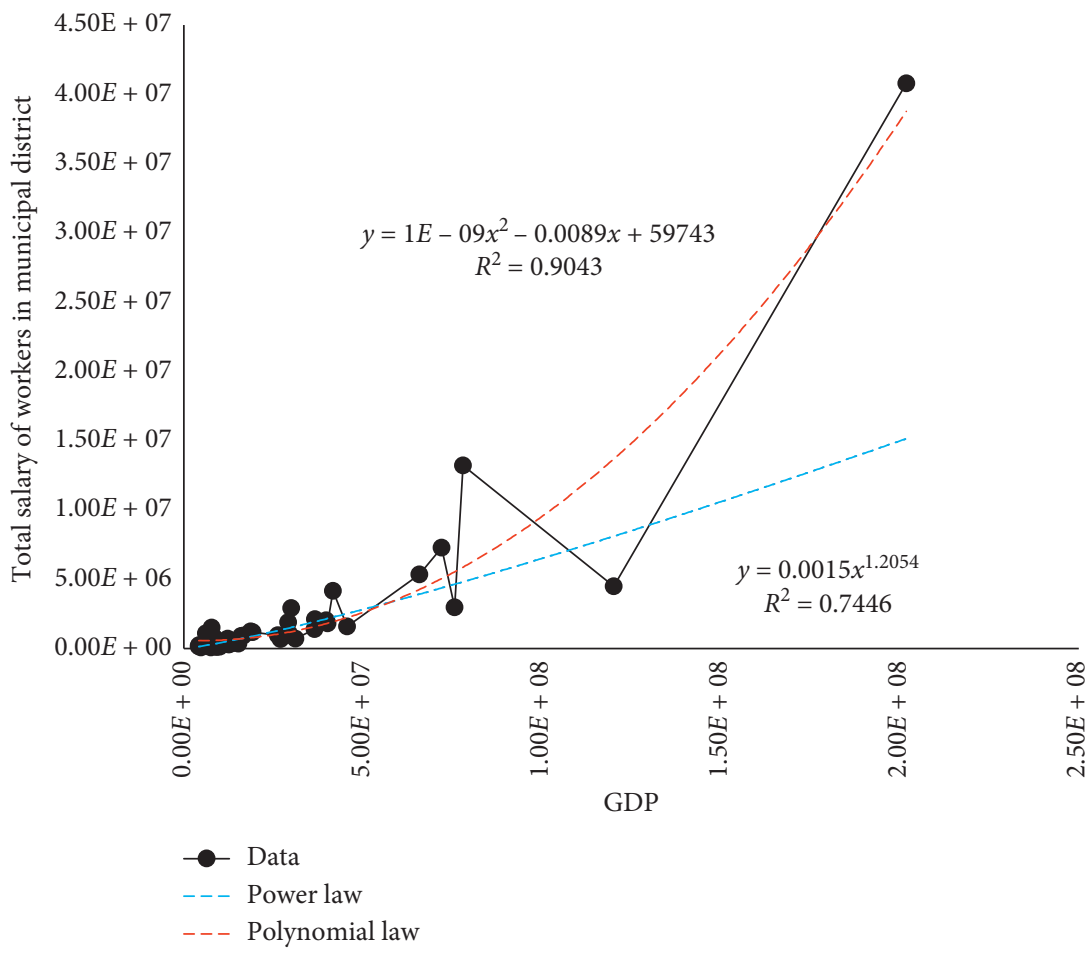

(b)

FIgURE 3: Allometric growth analysis. (a) Top 45 prefecture-level cities' GDP-area. (b) 52 prefecture-level cities GDP-total salary of workers in municipal district.

(3) In the case of sorting data in descending order, the information dimension (0.5834) and the correlation dimension $(-0.0308$ is an estimated figure, and the error is ignored. Its topological dimension is set as 0 ) of the GDP per capita (on the macroscale level) have the lowest values. This result indicates that the agglomeration of regional macroscale activities is obvious and that prefecture-level cities are more 

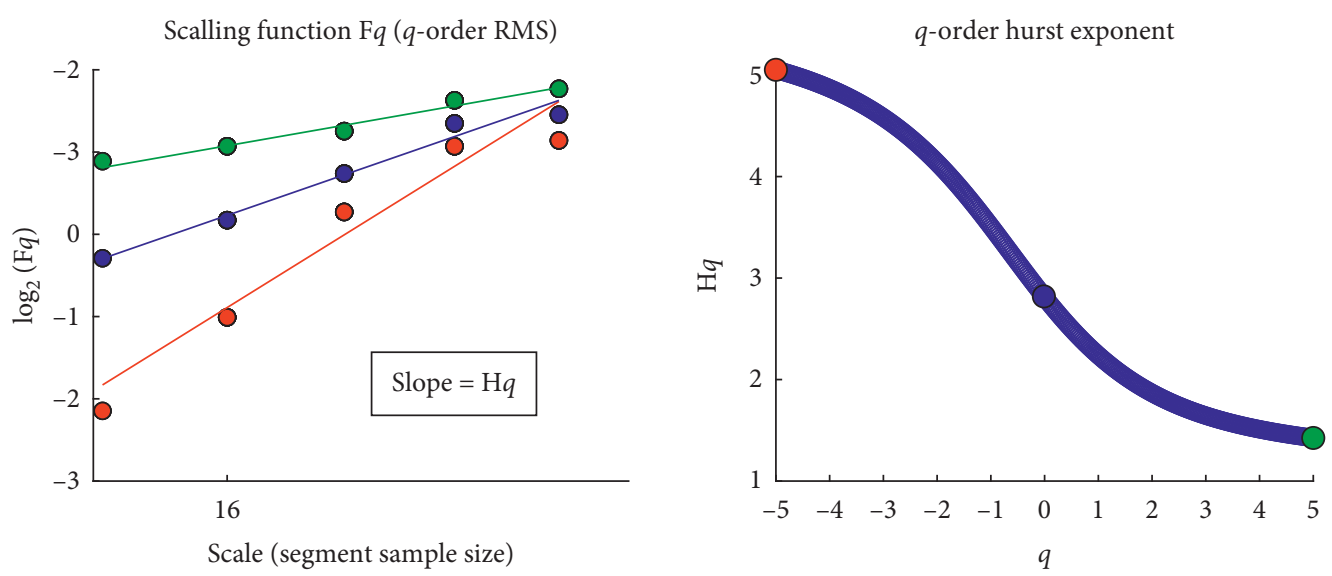
ㅇ $q=-5$
$q=-5$
- $q=-0.013193$
$q=-0.013193$
○ $q=5$
$q=5$

O Data1

$\mathrm{H} q(-5)=2.9109$

- $\mathrm{H} q(-0.013193)=2.8194$

$\mathrm{H} q(5)=1.4249$
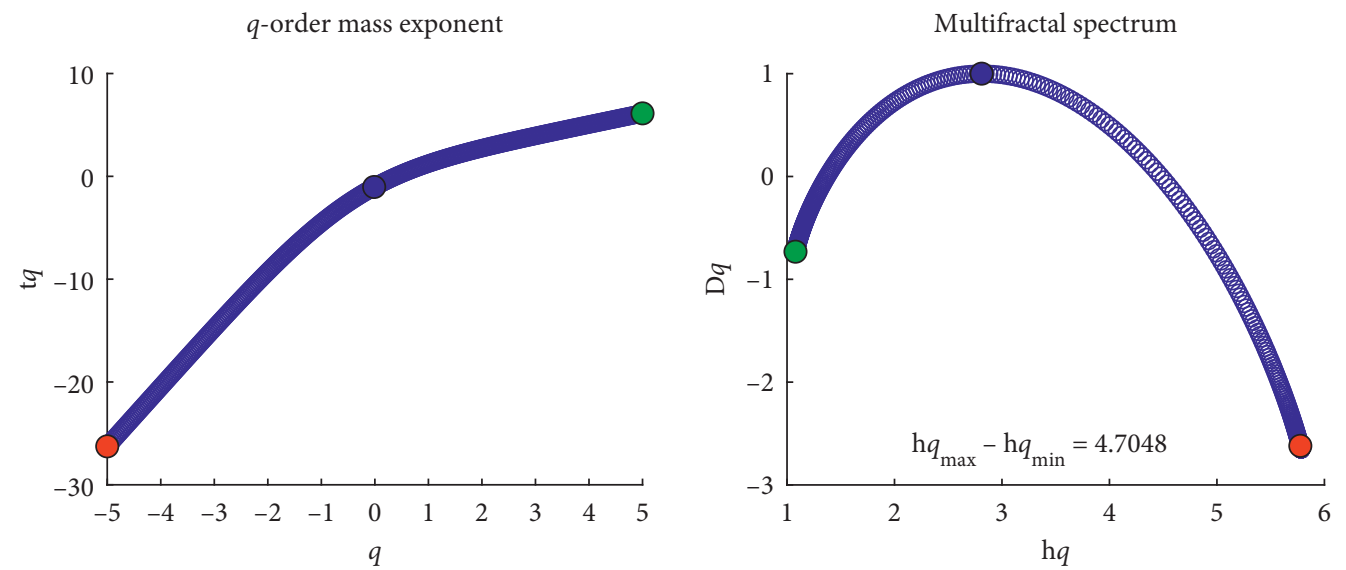
O Data1
$\mathrm{t} q(5)=6.1247$
$\mathrm{H} q(-5)=5.0521$
- $\mathrm{H} q(-0.013193)=2.8194$
O Data1
$\mathrm{D} q(-5)=-2.6197 \mathrm{~h} q(-5)=5.776$
- $\mathrm{D} q(-0.013193)=1.0001$ $\mathrm{h} q(-0.013193)=2.8107$
$\mathrm{D} q(5)=-0.73149 \mathrm{~h} q(5)=1.0786$

(a)

FIgUre 4: Continued. 

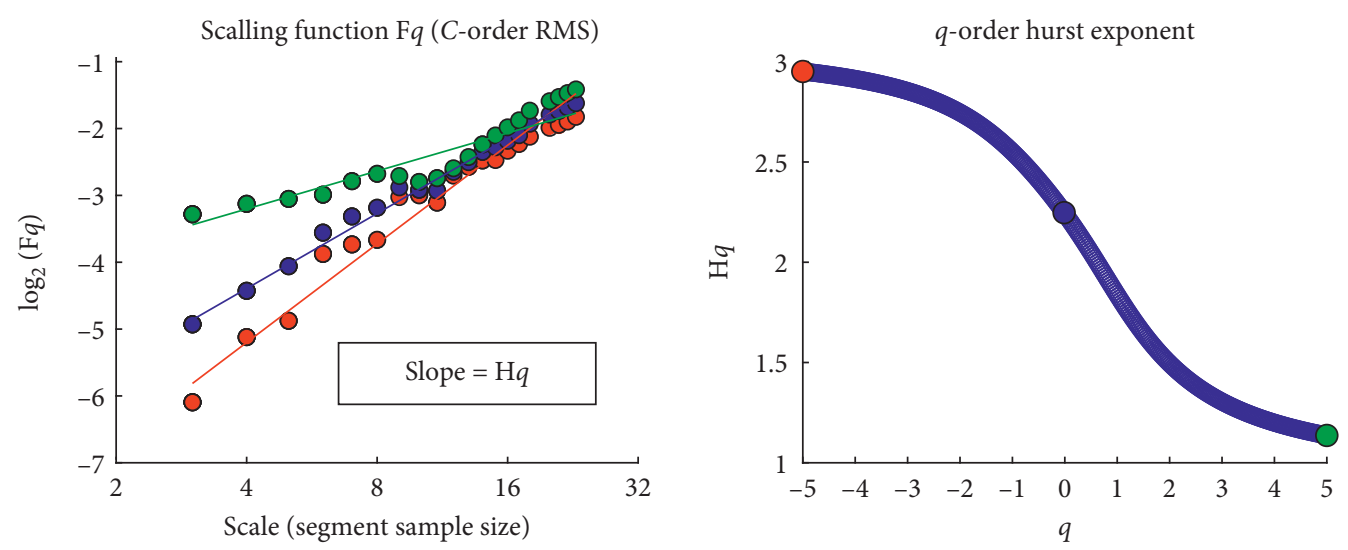
- $q=-5$
$q=-5$
- $q=-0.013193-q=-0.013193$
○ $q=5$
$q=5$

O Datal

$\mathrm{H} q(-5)=5.0521$

- $\mathrm{H} q(-0.013193)=2.2472$

$\mathrm{H} q(5)=1.1363$
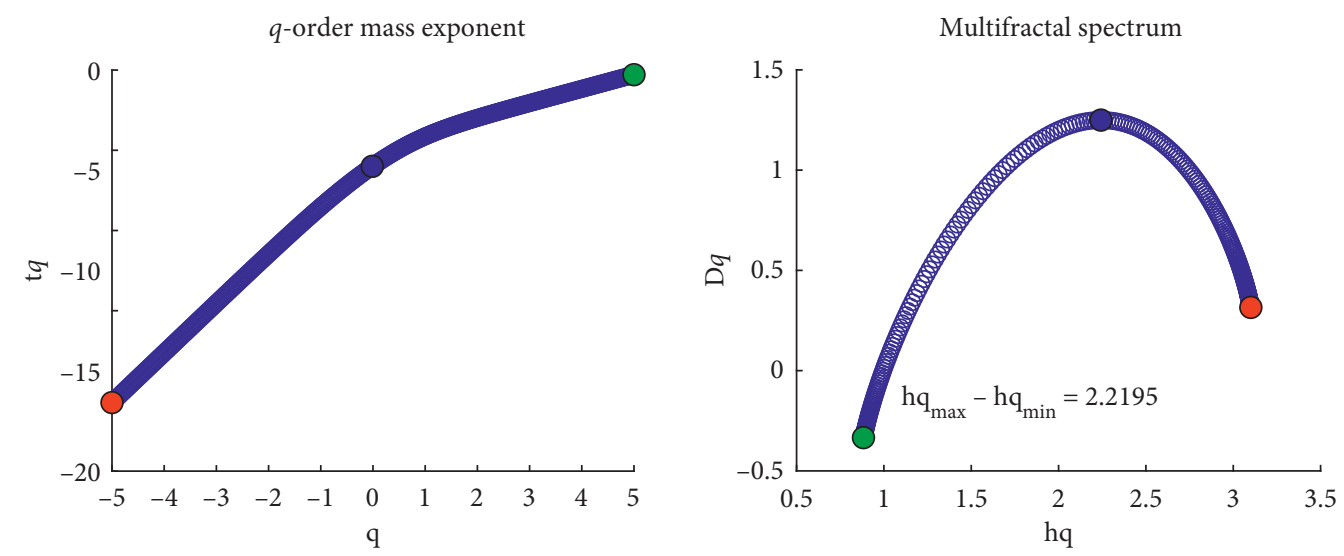
O Data1
$\mathrm{t} q(5)=4.6815$
$\mathrm{H} q(-5)=2.9513$
$\mathrm{H} q(-0.013193)=2.2472$
O Datal
$\mathrm{D} q(-5)=0.25274 \mathrm{~h} q(-5)=3.1008$
- $\mathrm{D} q(-0.013193)=1.0001$ $\mathrm{h} q(-0.013193)=2.2424$
○ $\mathrm{D} q(5)=0.2667 \mathrm{~h} q^{\prime}(5)=0.88296$

(b)

Figure 4: Continued. 

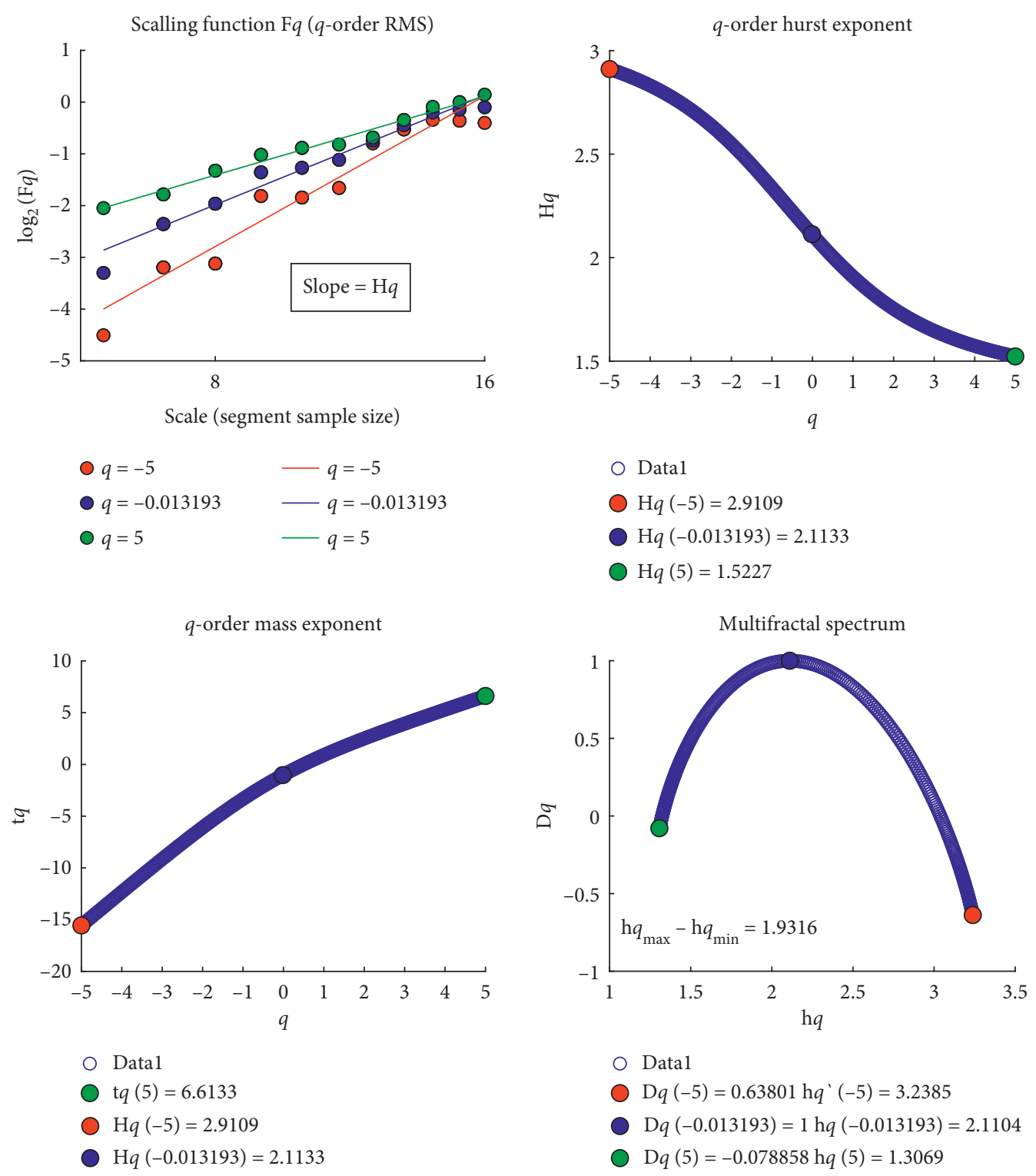

(c)

FIGURE 4: Illustration of the multifractal analysis for four provinces and one city in the lower reaches of the Yangtze River. (a) Illustration of the multifractal analysis of the scale of GDP. (b) Illustration of the multifractal analysis of the GDP per capita. (c) Illustration of the multifractal analysis of the average salary in the municipal districts.

TABLE 3: Results of the multifractal analysis for the four indicators of four provinces and one city in the lower reaches of the YangtzeRiver.

\begin{tabular}{lcccc}
\hline & GDP & GDP per capita & Average salary in the municipal districts & Built-up area \\
\hline Capacity dimension $\left(D_{0}\right)$ & 1.000945 & 1.000063 & 1.000038 & $1.000032^{*}$ \\
Information dimension $\left(D_{1}\right)$ & 0.702946 & 0.583352 & 0.81852 & $0.776306^{*}$ \\
Correlation dimension $\left(D_{2}\right)$ & 0.147988 & $-0.03077^{* *}$ & 0.482637 & $0.499892^{*}$ \\
\hline
\end{tabular}

${ }^{*}$ The primary data is normalised; ${ }^{* *}$ this is an estimated value, and the error is ignored.

independent. Meanwhile, the levels of the macroscale development of the 52 prefecture-level cities are different; in particular, the largest scale of macroscale development transformation is from the cores of economic development (such as Suzhou, Wuxi, and other cities) to the small- and medium-sized cities.
This phenomenon reflects an accumulation of the polarisation effects from the allometric growth in various economic activities. The allometric growth in various economic activities is also the reason behind the hierarchical GDP development in the 52 cities. The pattern of GDP development is not complex 
because the value of its multifractal spectrum width (2.2195) is between the multifractal spectrum widths of the indicators that were established in points 2 and 3.

(4) The built-up areas in the 52 prefecture-level cities (on the mesoscale level) present multifractal features by using normalised data. The parameters of the converted fractal spectrum are not comparable to the parameters of the above indicators, but they are consistent from a theoretical perspective. The implied regularity reflects that the hierarchical evolution of this indicator is more complex.

(5) When compared to the truncation of the multifractal dimension spectrum of the indicators of the GDP per capita and built-up area, the values of the GDP and the average salary in the municipal districts indicate that the driving force (salary) of the comprehensive regional development and the microscale activities is oriented to large- and medium-sized cities. Correspondingly, the values of the regional GDP per capita and built-up area (in particular, the values of the built-up area are truncated more) reflect the effects on regional development of the national balance strategy and "excessive urbanisation" in small- and medium-sized cities.

The hierarchical multifractal values of the comprehensive indicator and micro-meso-macro-scale indicators (calculated with data sorted in descending order) objectively reflect their evolutionary intensities and complexities in the regional development. The preceding analysis helps to clarify the details of regional multifields evolution; accordingly, one can derive the evolutionary mechanism of each field. The external manifestation of the mechanism is the spatial autocorrelation that is shown in Tables 2 and 3 and Figure 2 (Section 2.1).

4.2. Development of Preliminary Understanding. Section 2 is concerned with the external law regarding the spatial changes in the lower reaches of the Yangtze River, and Section 3.1 analyses the internal mechanism and details of the spatial evolution in four typical fields. Drawing on the findings in these two sections, one can develop a more profound understanding than that in Section 2.3:

(1) There are different patterns of spatial autocorrelation phenomena in the micro-meso-macro-scale fields in the lower reaches of the Yangtze River. The combination of these autocorrelations generates different urban-rural integration modes and thus promotes the development of the entire region. However, because of varied self-organisation, the development in the periphery is not as advanced as the development in the Yangtze River Delta. First, Anhui, Jiangxi, and the north of Jiangsu Province (which correspond to the $6^{\text {th }}$ and $4^{\text {th }}$ modes in Section 2.3) are not fully involved in the "Greater Shanghai Economic Circle." Second, the spatial autocorrelation of the regional distributions of the $2^{\text {nd }}, 3^{\text {rd }}$, and $5^{\text {th }}$ modes indicate that the development in the lower reaches of the Yangtze River is complex and uncoordinated.

(2) Under the influence of many factors, the hierarchical evolution in the above four fields tends to follow the multifractal law, but the numerical hierarchical fractal is different from the spatial fractal. For this reason, this research adopts only three indicators (which were selected in Section 2.2) to analyse the entire region. Specifically, these three indicators were adopted because the evolutions in the above three fields satisfy the conditions of vertical hierarchical multifractal and horizontal spatial autocorrelation. The GDP does not meet some requirements of spatial autocorrelation, but it is adopted in this research because it provides much information concerning the regional micro-meso-macro-scale development.

(3) Government intervention has resulted in the "excessive urbanisation" of the urban built-up area and the homogenisation of the average salary. These phenomena show that the multifields evolution on the micro-meso-macro-scale levels in the lower reaches of the Yangtze River experiences allometric growth and generates multifractal features, and there are a different evolution mechanisms and features in different fields.

(4) The multifractal features and trends of evolution in different fields will produce many uncoordinated and unsynchronised phenomena (collectively called multiple spatial dissymmetry). Multiple spatial dissymmetry has been the normal state of development in the four provinces and one city in the lower reaches of the Yangtze River since the reform and opening up.

\section{Multiple Spatial Dissymmetry: Analysis of Regional Allometric Growth}

Sections 2 and 3 are concerned with analysing and demonstrating the heterogeneity and complexity of the spatial development and hierarchical evolution in the lower reaches of the Yangtze River. The reason for this heterogeneity and complexity is allometric growth in various fields. When considering the impact of allometric growth, Section 4.1 explores the "maximum common divisor" of regional allometric growth, and Section 4.2 analyses the spatial differences of allometric growth and conducts an in-depth analysis for multifields spatial dissymmetry.

5.1. Maximum Common Divisor of Allometric Growth. This paper adopts the entropy weighting method to analyse the GDP per capita, built-up area, and average salary in the municipal district with cross-sectional data from 2012. The results show that the weighting factors of the three indicators are $0.2646,0.5748$, and 0.1606 , respectively, and this paper calculates the comprehensive development level of 52 prefecture-level cities with these data (Figure 5). The analysis 


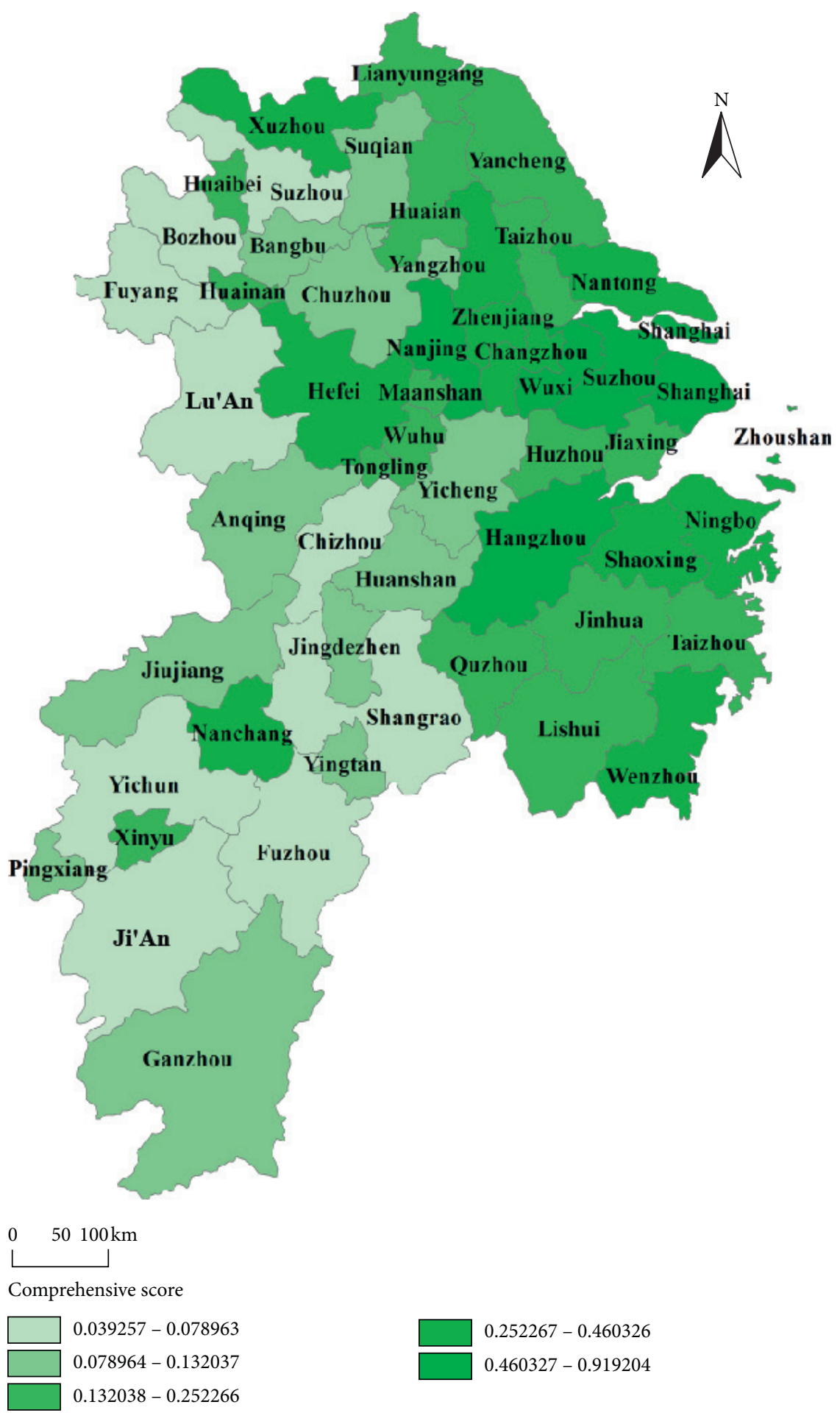

Figure 5: Comprehensive ratings of the urban-rural integration in the lower reaches of the Yangtze river in 2012.

shows the following: (1) The comprehensive rating of urbanrural development in the area that comprises Shanghai, Suzhou, Wuxi (at the peripheral area of Shanghai), Nanjing, and Hangzhou (two capital cities) is the highest. (2) The comprehensive rating of urban-rural development in the eastern coastal area (the Yangtze River Delta and the entire area in the provinces of Zhejiang and Jiangsu) is high. (3) The comprehensive rating of urban-rural development in the central and western areas is not high, but the capital cities and the cities in the centre of this region (such as Xuzhou and Wenzhou) are important for promoting urban-rural development. When considering the entire region, one can argue that (1) the built-up areas (towns) dominate the urban-rural integration of the region and of the prefecturelevel cities because more than $50 \%$ of the information is collected from urban built-up areas and (2) from the 
perspective of allometric growth, the attributes of the urban area diffuse to the countryside. This diffusion induces dissymmetry on the micro-meso-macro-scale level and produces various modes of urban-rural integration.

Figure 5 reflects the comprehensive evaluation of the quantification of the lower reaches of the Yangtze River and actually reflects the "maximum common divisor" of the allometric growth in this region. Accordingly, Figure 5 illustrates the spatial expansion pattern of the regional development. Based on the "maximum common divisor," multifields allometric growth and comprehensive spatial complexity emerge in the 52 prefecture-level cities.

\subsection{Exploration of the Essence of Multiple Spatial} Dissymmetry. Table 2 and Figure 2, which are generated from the spatial autocorrelation analysis, show the details of external space using examples of three typical micro-mesomacro-scale indicators. These indicators essentially demonstrate the results of the evolution in each field, and each field evolves on the corresponding allometric growth rate, which is produced by the "maximum common divisor." Based on the preceding findings, this research discloses the multifields spatial dissymmetry that results from allometric growth by comparing the hierarchical data of the average salary, GDP per capita, and built-up area of 52 prefecturelevel cities. The fitting curves show that they are all within the three scale ranges $[33,34]$. Almost all the large cities and some prefecture-level cities (the values of the corresponding indicators are ranked at the same positions on the sequence) are within the first range, and the development within this range cannot represent the overall development in the lower reaches of the Yangtze River. Small- and medium-sized prefecture-level cities that are surrounded by the underdeveloped peripheral area are within the third range, and the development within this range similarly cannot represent the development in the entire region. Within the second range, there are more prefecture-level cities, and the corresponding indicators are ranked differently on the sequence (Table 4 and Figure 6); this development can represent the development of the entire region. Therefore, this paper conducts an analysis of the regional development by drawing on the development within the second scale range.

\subsubsection{Sequences of the Built-Up Areas and Average Salary in} the Municipal Districts (Meso-Micro-Scale Spatial Dissymmetry). Figure 6(a) shows that there is a significant dissymmetry between the sequences of built-up areas and the average salary in the municipal districts. The most significant positive dissymmetry (the former indicator ranked higher on the sequence than the latter indicator; negative dissymmetry occurs when the latter indicator ranks higher than the former indicator) is in an area, and there are 2-4 cities between this area and Shanghai. This finding indicates that the area that includes the south of Jiangsu Province mode (the $2^{\text {nd }}$ mode in Section 2.3) and the transitional mode (the $4^{\text {th }}$ mode in Section 2.3) is developing quickly; the rapid expansion of this built-up area probably results from "excessive urbanisation." The negative dissymmetry can be found in the peripheral area that includes the $6^{\text {th }}$ mode (Section 2.3); the reason for the significant dissymmetry in the northwest of Anhui Province is that Huainan, Huaibei, and Haozhou are mining cities that create exceptions. Generally, the spatial dissymmetry results from different rates of allometric growth in the meso-microscale typical fields in prefecture-level cities.

Figure 6(a) shows that Shanghai and the Yangtze River Delta have formed a stable polarised centre and that the peripheral area is the hinterland for development; mesomicro-scale dissymmetry is mainly found in the central region (primarily within the second scale range, sic passim). The various self-organisation changes (multifractal) in the two fields result in allometric growth in the region and eventually affect the evolution of the six urban-rural integration modes. This meso-micro-scale development represents the basic pattern of the development in the lower reaches of the Yangtze River.

\subsubsection{Sequences of the Built-Up Areas and GDP per Capita} (Meso-Macro-Scale Spatial Dissymmetry). Figure 6(b) shows that the two indicators have more significant spatial polarisation-diffusion effects; one can easily find positive dissymmetry in the cities that agglomerate towards Shanghai (the $1^{\text {st }}$ mode in Section 2.3). Negative dissymmetry can be easily found in the peripheral area that includes the traditional mode (Section 2.3), and the most significant negative dissymmetry is in Anhui Province. The dissymmetry in prefecture-level cities that include the transitional mode in the central area (the $4^{\text {th }}$ mode in Section 2.3) is similar; the negative dissymmetry found in Wenzhou discloses the uniqueness of the Wenzhou mode (the $3^{\text {rd }}$ mode in Section 2.3). In general, there is regular allometric growth in these two meso-macro-scale fields, and it dominates the development of the region.

By comparing Figures 6(a) and 6(b), one can easily find the "excessive urbanisation" phenomenon in the middle and eastern part of the region, which explains the statistical insignificance of the indicator of built-up areas in the spatial autocorrelation analysis.

\subsubsection{Sequences of the Average Salary in the Municipal} Districts and GDP per Capita (Micro-Macro-Scale Spatial Dissymmetry). Figure 6(c) shows that there is no regularity between the two indicators, and, as a matter of experience, this should be the result of government regulation. Specifically, the market economy will widen the gaps between grassroots production and living activities; therefore, the irregularity between these two indicators is the result of government regulation aiming to promote urban-rural integration and narrow the urban-rural gap. However, this topic is beyond the scope of this research.

Therefore, the allometric growth with the "maximum common divisor" (as shown in Figure 5), the allometric growth rate, and the consistent/inconsistent process of various allometric growth are the reasons behind the complex evolution and multiple spatial dissymmetry in the various fields in the lower reaches of the Yangtze River. 


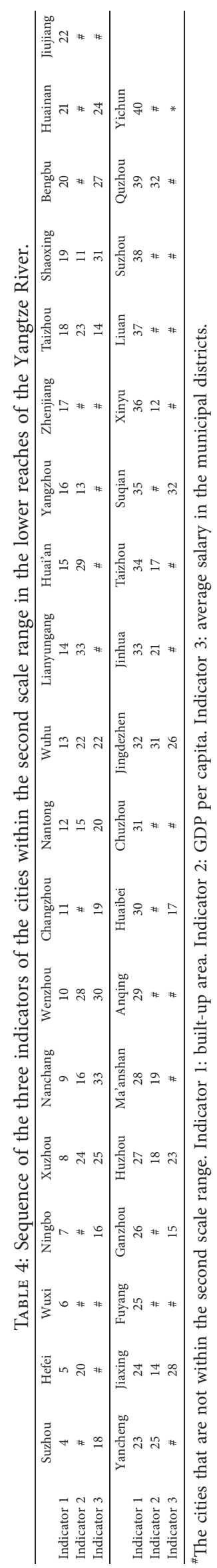




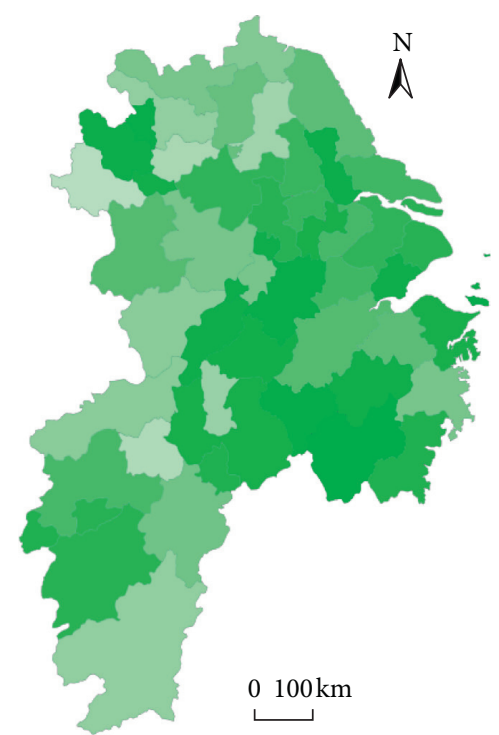

(a)

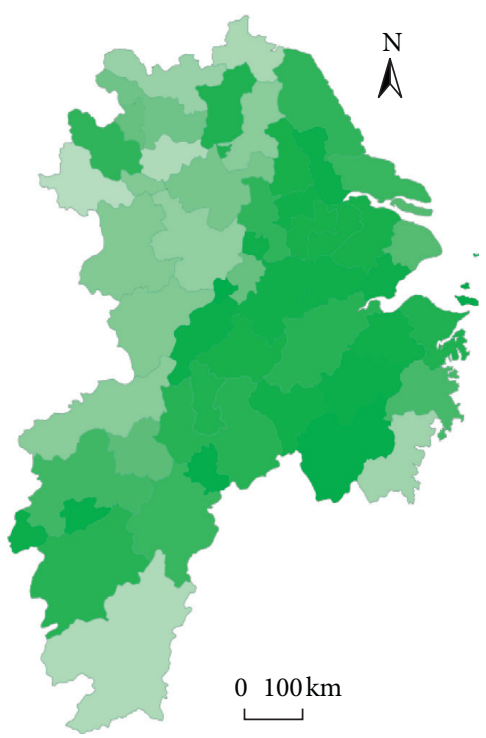

(b)

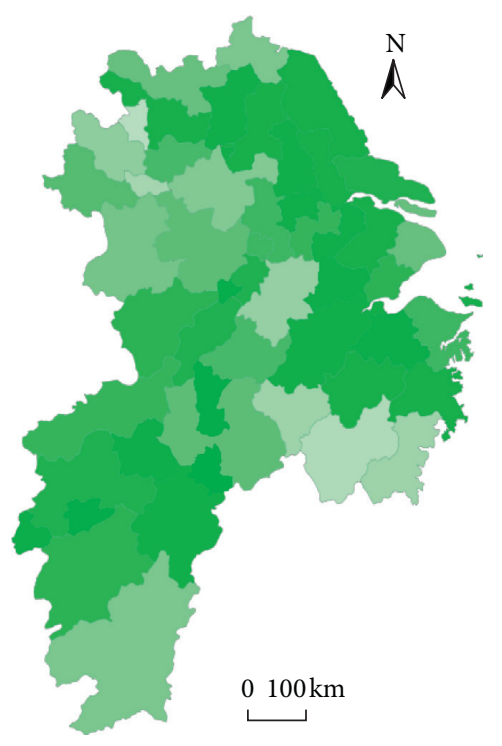

(c)

Figure 6: Illustration of the spatial dissymmetry of four provinces and one city in the lower reaches of the Yangtze river (darker colour indicates greater positive differences).

Compared to previous studies concerning this arena, this research contributes to a more advanced analytical perspective and a quantitative method by adopting spatial autocorrelation (Section 2.2) and multifractal (Section 3.2) analyses.

\section{Discussion}

For a complex spatial region, its multidomain external spatial autocorrelation, internal allometric growth, and real multifractal features are interrelated. Therefore, only a comprehensive analysis based on three types of analysis can lead to a scientific conclusion. For the lower reaches of the Yangtze River, this article uses the above three methods to conduct case studies of its multifield development. Its main conclusions are as follows.

\subsection{Regional Development and Urban-Rural Integration.} The allometric growth in various fields and the integration in prefecture-level cities in the lower reaches of the Yangtze River form a differentiated spatial layout of urban-oriented urban-rural integration. The regional evolution mode that comprises the micro-meso-macro-scale multilevel coupling can be divided into six categories: (1) Shanghai's urbanisation mode, (2) the Yangtze River Delta mode with a higher level of development (the south of Jiangsu Province mode), (3) the unique development mode of the south of Zhejiang Province (Wenzhou mode), (4) the transitional mode in the central region, (5) the polarisation mode in the central region, and (6) the traditional mode.

6.2. Multifields Allometric Growth and Multifractal Features. Although the development in the lower reaches of the Yangtze River has become a network (i.e., a spatial multifractal evolution), it still has the overall spatial polarisation and diffusion effect. Specifically, the overall development of the region is dominated by urban attributes and tends to follow power law evolution; thus, the development level of the region-from Yangtze River Delta to the peripheral area-is declining at an anisotropy/nonequal ratio.

6.3. Spatial Complexity of Urban-Rural Development. It can find allometric growth in the microscale activities, medium patterns, and macroscale development in the lower reaches of the Yangtze River, and they all have three scale ranges. However, analysis shows that the development in metropolises (such as Shanghai, Nanjing, and Hangzhou within the first scale range) and in the small- and medium-sized cities (within the third scale range) cannot represent the development of the entire region, while the development in the cities within the second scale range can represent the typical development of the lower reaches of the Yangtze River. In addition, the allometric growth in various fields generates the complexity of different intensities. The spectrum width of the multifractal spectrum shows that the comprehensive development of prefecture-level cities is more complex (spectrum width is 4.7048), the regional macroeconomic activities (2.2195) are affected by the industrial layout, and the salary-oriented microscale activities (1.9316) are simpler. Currently, the expansion in built-up areas is irregular; although there are multifractal features, one can find "excessive urbanisation" in the medium- and small-sized cities.

\section{Conclusion and Future Works}

These modes infiltrate and interact with one another and generate the characteristics of self-organising features (e.g., 
multifractal) in some fields. Nevertheless, the self-organisation development of the Yangtze River Delta has not yet fully covered the peripheral area of the region, and the distribution areas of the $4^{\text {th }}$ and the $6^{\text {th }}$ modes have not become fully involved in the "Greater Shanghai economic circle." In addition, the distribution area of modes 2 to 5 discloses the complexity of the development in the lower reaches of the Yangtze River and the incongruity in the various fields. Therefore, the comprehensive development and microscale activities of the 52 prefecture-level cities tend to show allometric growth that is oriented to large- and medium-sized cities. The macro-meso-scale dimensional activities tend to have allometric growth that is oriented to medium- and small-sized cities, which reflects the impact of the national balance strategy and the status of "excessive urbanisation" in the medium- and small-sized cities.

Based on the preceding findings, corresponding evolution modes of the development and spatial coupling status have emerged in many fields. Allometric growth is often manifested in the form of the weakening of other functions or power functions, produces many spatial hierarchical multifractal features, and then affects the basic layout of the urban-rural development. Therefore, the development in various fields has produced detailed development differences and causes many problems in urban-rural development (e.g., multiple spatial dissymmetry). Accordingly, the seemingly chaotic process of urban-rural development in the lower reaches of the Yangtze River has followed the evolution law, which has certain spatial autocorrelation and hierarchical multifractal features. A quantitative understanding and graphical representation that were generated from spatial autocorrelation (Section 2.2) and multifractals (Section 3.2) help us to gain insight into the allometric growth and complex changes in the lower reaches of the Yangtze River. The understanding of the allometric growth and multifractal characteristics of the regional space has been achieved. However, in reality, we combine intuitive spatial autocorrelation analysis with partial theoretic allometric analysis and multifractal analysis, which is conducive to the formation of a global understanding of the overall and detailed spatial changes in the region.

In a specific analysis, geographical studies generally determine the scope of the study area. For the general allometric cross-sectional analysis, it is not necessary to determine the spatial scope of each development field one by one, because the relevant workload is large and the development of multiple fields is more in the evolution process and does not reach the ideal end. The range of allocating differently in different fields is generally not the same. In addition, in this case study, the development of multiple areas in the lower reaches of the Yangtze River should actually be a comprehensive analysis of longitudinal time series + horizontal spatial sequences. In this paper, only oneyear cross-sectional data has been selected for analysis. Obviously, the analysis of supportability of time series is not enough, so the selection of one-year indicators is very random.

Our future research will focus on the following aspects: (1) the implementation of artificial intelligence tools, such as computational intelligence assisted design framework [35-37], to reveal urban/suburban change; (2) the development of operational strategy of highly efficient urban study using big data; (3) high-efficiency visualisation tools to demonstrate the multifractal features, such as augmented reality/virtual reality techniques.

\section{Data Availability}

The data that support the findings of this study are available from the first author, Zhi-jun Song, or the corresponding author, Yi Chen, upon reasonable request.

\section{Conflicts of Interest}

The authors declare that they have no conflicts of interest.

\section{Authors' Contributions}

Zhi-jun Song and Yi Chen conceived of the presented idea, developed the theory, and performed the computations. Yun $\mathrm{Li}$ and Xin Zhao verified the analytical methods. Yun Li supervised the findings of this work. All authors processed the experimental data, performed the analysis, drafted the manuscript, discussed the results, and contributed to the final manuscript.

\section{Acknowledgments}

The research was supported by a Key Project from $\mathrm{Hu}-$ manities and Social Science Research Institutes in Ministry of Education of China (16JJD770021) and the Dongguan University of Technology under research grant Industry 4.0 Smart Design and Innovation Platform (no. KCYXM2017012).

\section{References}

[1] X. Duan, X. Yu, and J. Nipper, "Study on the expanded scope of the Yangtze delta region based on the function of economic polarized area," Acta Geographica Sinica, vol. 64, no. 2, pp. 211-220, 2009, in Chinese.

[2] C. Jin and Y. Q. Lu, "Research on evolvement of spatial pattern of economy in Yangtze river delta since 1978," Human Geography, no. 2, pp. 113-118, 2012, in Chinese.

[3] Q. Xu, K. J. Yang, and Y. Z. Huang, "Types, evolution and healthy development countermeasure of rural space in waternet area of Yangtze river," Research of Agricultural Modernization, vol. 33, no. 3, pp. 336-340, 2012, in Chinese.

[4] P. Zhao, "Too complex to be managed? new trends in periurbanisation and its planning in Beijing," Cities, vol. 30, pp. 68-76, 2013.

[5] Y. Yang and C. F. Wu, "Regional economic disparities in panYangtze river delta-based on EBI and EBIi," Resources and Environment in the Yangtze Basin, vol. 20, no. 5, pp. 513-518, 2011, in Chinese.

[6] J. N. Wu, S. M. Yao, and Y. H. Cao, "Differentiation of urban and rural harmonious development in the Yangtze river delta urban agglomeration," Resources and Environment in the Yangtze Basin, vol. 19, no. S1, pp. 21-26, 2010, in Chinese. 
[7] S. H. Xie and L. X. Wang, "The urban-rural development's coordination degree evaluation of 16 cities in Yangtze river delta," Urban Problems, no. 10, pp. 63-67, 2013, in Chinese.

[8] Q. Che, X. Duan, and Y. Guo, "Urban spatial expansion process, pattern and mechanism in Yangtze river delta," Acta Geographica Sinica, vol. 66, no. 4, pp. 446-456, 2011, in Chinese.

[9] G. Tian, J. Jiang, Z. Yang, and Y. Zhang, "The urban growth, size distribution and spatio-temporal dynamic pattern of the Yangtze river delta megalopolitan region, China," Ecological Modelling, vol. 222, no. 3, pp. 865-878, 2011.

[10] B. Zhou, T. Dai, and J. Liang, "The evolution of the level of economic scale of urban system based on fractal analysis," Scientia Geographica Sinica, vol. 32, no. 2, pp. 156-162, 2012, in Chinese.

[11] J.-X. Wu, X. Cheng, H.-S. Xiao, H. Wang, L.-Z. Yang, and E. C. Ellis, "Agricultural landscape change in China's Yangtze delta, 1942-2002: a case study," Agriculture, Ecosystems \& Environment, vol. 129, no. 4, pp. 523-533, 2009.

[12] L. Zhang and Y. Q. Lu, "Studies on spatial analysis method of the "pole \& axis system": a case study of the Yangtze river delta," Acta Geographica Sinica, vol. 65, no. 12, pp. 1534-1547, 2010, in Chinese.

[13] Y. Chen and J. Wang, "Multifractal characterization of urban form and growth: the case of Beijing," Environment and Planning B: Planning and Design, vol. 40, no. 5, pp. 884-904, 2013.

[14] J. Wang, W. Chen, and F. Yuan, "Human mobility and evolution based on social network: an empirical analysis of Yangtze river delta," Geographical Research, vol. 33, no. 2, pp. 385-400, 2014, in Chinese.

[15] W. Chen and J. Wang, "Assessment and measurement of spatial integration in the Changjiang river delta," Scientia Geographica Sinica, vol. 33, no. 8, pp. 902-908, 2016, in Chinese.

[16] Y. Chen and S. Jiang, "An analytical process of the spatiotemporal evolution of urban systems based on allometric and fractal ideas," Chaos Solitons \& Fractals, vol. 39, no. 1, pp. 49-64, 2009.

[17] Y. Chen, "Multi-scaling allometric analysis for urban and regional development," Physica A: Statistical Mechanics and Its Applications, vol. 465, pp. 673-689, 2017.

[18] J.-H. He and Z. Huang, "A novel model for allometric scaling laws for different organs," Chaos, Solitons \& Fractals, vol. 27, no. 4, pp. 1108-1114, 2006.

[19] B. J. West and L. Griffin, "Allometric control, inverse power laws and human gait," Chaos, Solitons \& Fractals, vol. 10, no. 9, pp. 1519-1527, 1999.

[20] Y. G. Chen, Fractal Urban Systems: Scaling, Symmetry and Spatial Complexity, Science Press, Beijing, China, 2008, in Chinese.

[21] E. A. F. Ihlen, "Introduction to multifractal detrended fluctuation analysis in matlab," Frontiers in Physiology, vol. 3, 2012.

[22] J. W. Kantelhardt, S. A. Zschiegner, E. Koscielny-Bunde, S. Havlin, A. Bunde, and H. E. Stanley, "Multifractal detrended fluctuation analysis of nonstationary time series," Physica A: Statistical Mechanics and Its Applications, vol. 316, no. 1-4, pp. 87-114, 2002.

[23] Y. F. Mu, "Evolution and coupling of industry and employment in Yangtze river delta," Economic Geography, vol. 33, no. 10, pp. 95-102, 2013, in Chinese.

[24] The National Bureau of Statistics, 2013 China City Statistical Yearbook for Regional Economy, China Statistics Press, Beijing, China, 2013, in Chinese.
[25] The Population and Employment Statistics Division of National Bureau of Statistics, China Statistical Yearbook for Regional Economy-2013, China Statistics Press, Beijing, China, 2013, in Chinese.

[26] Y. Chen, "Derivation of the functional relations between fractal dimension of and shape indices of urban form," Computers, Environment and Urban Systems, vol. 35, no. 6, pp. 442-451, 2011.

[27] Z. J. Song and L. M. Liu, "Allometric Scaling of Multiple Development Functions of Beijing from 1979-2009," Economic Geography, vol. 36, no. 1, pp. 53-60, 2016, in Chinese.

[28] J. Bai, "On regional innovation efficiency: evidence from panel data of China's different provinces," Regional Studies, vol. 47, no. 5, pp. 773-788, 2013.

[29] Y. Liu, R. Yang, and Y. Li, "Potential of land consolidation of hollowed villages under different urbanization scenarios in China," Journal of Geographical Sciences, vol. 23, no. 3, pp. 503-512, 2013.

[30] H. Long, "Land consolidation: an indispensable way of spatial restructuring in rural China," Journal of Geographical Sciences, vol. 24, no. 2, pp. 211-225, 2014.

[31] J. X. Zhang, X. L. Luo, and J. Yin, "Polycentric mega-city regions and multi-level governance of the Yangtze river delta," Urban Planning International, vol. 23, no. 1, pp. 65-69, 2008, in Chinese.

[32] A. Rényi, "On measures of information and entropy," in Proceedings of the Fourth Berkeley Symposium on Mathematics Statistics and Probability, pp. 547-561, Los Angeles, CL, USA, 1961.

[33] Y. G. Chen, "Modeing fractal structure of city-size distributions using correlation functions," PloS One, vol. 6, no. 9, Article ID e24791, 2011.

[34] L.-S. Huang and Y.-G. Chen, "A comparison between two OLS-based approaches to estimating urban multifractal parameters," Fractals, vol. 26, no. 01, p. 1850019, 2018.

[35] Y. Chen, Z. Song, G. Zhang, M. T. Majeed, and Y. Li, "Spatiotemporal evolutionary analysis of the township enterprises of beijing suburbs using computational intelligence assisted design framework," Palgrave Communications, vol. 4, no. 1, 2018.

[36] Y. Chen and Y. Li, Computational Intelligence Assisted Design (In the Era of Industry 4.0), CRC Press, Boca Raton, FL, USA, 2018.

[37] Y. Chen and Y. Li, "Intelligent autonomous pollination for future farming-a micro air vehicle conceptual framework with artificial intelligence and human-in-the-loop," IEEE Access, vol. 7, no. 1, pp. 119706-119717, 2019. 